\title{
Article
}

\section{DIRECT CONFIRMATION OF TWO PATTERN SPEEDS IN THE DOUBLE-BARRED GALAXY NGC 29501}

Corsini, E M, Debattista, Victor P and Aguerri, J A L Available at https://clok.uclan.ac.uk/17009/

Corsini, E M, Debattista, Victor P orcid iconORCID: 0000-0001-7902-0116 and Aguerri, J A L (2003) DIRECT CONFIRMATION OF TWO PATTERN SPEEDS IN THE DOUBLE-BARRED GALAXY NGC 29501. The Astrophysical Journal, 599 . L29L32. ISSN 0004-637X

It is advisable to refer to the publisher's version if you intend to cite from the work.

For more information about UCLan's research in this area go to http://www.uclan.ac.uk/researchgroups/ and search for <name of research Group>.

For information about Research generally at UCLan please go to http://www.uclan.ac.uk/research/

All outputs in CLoK are protected by Intellectual Property Rights law, including Copyright law. Copyright, IPR and Moral Rights for the works on this site are retained by the individual authors and/or other copyright owners. Terms and conditions for use of this material are defined in the policies page.

\section{CLoK}

Central Lancashire online Knowledge www.clok.uclan.ac.uk

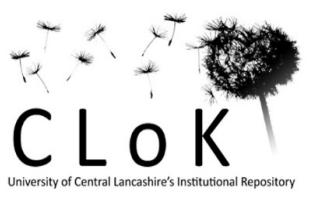




\title{
DIRECT CONFIRMATION OF TWO PATTERN SPEEDS IN THE DOUBLE-BARRED GALAXY NGC $2950^{1}$
}

\author{
E. M. Corsini, ${ }^{2}$ Victor P. Debattista, ${ }^{3}$ and J. A. L. Aguerri ${ }^{4}$ \\ Received 2003 September 15; accepted 2003 October 30; published 2003 November 21
}

\begin{abstract}
We present the surface photometry and stellar kinematics of NGC 2950, which is a nearby and undisturbed SB0 galaxy hosting two nested stellar bars. We use the Tremaine-Weinberg method to measure the pattern speed of the primary bar. This also permits us to establish directly and for the first time that the two nested bars are rotating with different pattern speeds and, in particular, that the rotation frequency of the secondary bar is higher than that of the primary one.
\end{abstract}

Subject headings: galaxies: elliptical and lenticular, cD — galaxies: individual (NGC 2950) — galaxies: kinematics and dynamics — galaxies: photometry — galaxies: structure

\section{INTRODUCTION}

Large-scale bars are present in some two-thirds of all disk galaxies (Knapen, Shlosman, \& Peletier 2000; Eskridge et al. 2000). Secondary stellar bars within large-scale bars are also common, occurring in about one-third of the barred galaxies (Laine et al. 2002; Erwin \& Sparke 2002). Interest in secondary stellar bars is motivated by the hypothesis that they are a mechanism for driving gas to small radii to feed the supermassive black holes powering active galactic nuclei (e.g., Regan \& Mulchaey 1999). However, the efficiency of such transport is uncertain because of our complete lack of knowledge of $\Omega_{p}$ and $\Omega_{s}$, the pattern speeds of the primary and secondary bars, respectively. Whereas a number of pattern speeds of large-scale bars have been measured (see Aguerri, Debattista, \& Corsini 2003, hereafter ADC03, and references therein), no such measurements in nested systems have been performed yet. The presence of nested bars with different pattern speeds has been inferred largely on the basis of their apparently random relative orientations (Buta \& Crocker 1993). The possibility that $\Omega_{s}>\Omega_{p}$ is supported by the simulations of Rautiainen, Salo, \& Laurikainen (2002), which showed that secondary bars can naturally form and survive for more than a few rotation periods in pure stellar disks. The morphological characteristics of these systems are suggestive of stars in the secondary bars oscillating about the loop orbits studied by Maciejewski \& Sparke (2000) for models with $\Omega_{s}>\Omega_{p}$. However, simulations have also found other possibilities (Friedli \& Martinet 1993), including cases in which two stellar bars counterrotate (Sellwood \& Merritt 1994; Friedli 1996).

A model-independent method for measuring pattern speeds is the Tremaine-Weinberg (TW) method (Tremaine \& Weinberg 1984). This gives the pattern speed $\Omega$ of a single bar as

$$
x \Omega \sin i=\mathcal{V},
$$

where $\chi$ and $\mathcal{V}$ are the luminosity-weighted average position

\footnotetext{
${ }^{1}$ Based on observations made with the UK Jacobus Kapteyn Telescope and the Italian Telescopio Nazionale Galileo operated at the Spanish Observatorio del Roque de los Muchachos of the Instituto de Astrofísica de Canarias by the Isaac Newton Group and the Istituto Nazionale di Astrofisica, respectively.

${ }^{2}$ Dipartimento di Astronomia, Università di Padova, Vicolo dell'Osservatorio 2, I-35122 Padova, Italy; corsini@pd.astro.it.

${ }^{3}$ Institut für Astronomie, ETH Hönggerberg, HPF G4.2, CH-8093 Zürich, Switzerland; debattis@phys.ethz.ch.

${ }^{4}$ Instituto de Astrofísica de Canarias, Vía Láctea s/n, E-38200 La Laguna, Tenerife, Canary Islands, Spain; jalfonso@11.iac.es.
}

and line-of-sight velocity measured parallel to the major axis of the galaxy disk, respectively, and $i$ is the disk inclination. Long-slit spectra parallel to the disk major axis can measure all the quantities needed by equation (1) provided that the galaxy is free of extinction. If several parallel slits at different offsets $Y$ relative to the major axis are available for a galaxy, $\Omega \sin i$ can be obtained as the slope of a plot of $\mathcal{V}$ versus $\mathcal{X}$. In a double-barred galaxy (hereafter S2B), for a slit passing through both bars, equation (1) is modified to

$$
\left(\chi_{p} \Omega_{p}+\chi_{s} \Omega_{s}\right) \sin i=\mathcal{V},
$$

provided the two bars are rigidly rotating through each other, i.e., that the total surface density is described by $\Sigma(R, \phi)=$ $\Sigma_{p}\left(R, \phi-\Omega_{p} t\right)+\Sigma_{s}\left(R, \phi-\Omega_{s} t\right)$. Equation (2) is then a consequence of the linearity of the continuity equation. Equation (2) can be solved for $\Omega_{s}$ by first measuring $\Omega_{p}$ as in equation (1) with slits that avoid the secondary bar and then by modeling to obtain $\chi_{s}$ from the observed $\chi=\chi_{s}+\chi_{p}$. However, the two bars are not likely to rotate rigidly through each other when $\Omega_{p} \neq \Omega_{s}$ (Louis \& Gerhard 1988; Maciejewski \& Sparke 2000; Rautiainen et al. 2002), requiring additional modeling to obtain $\Omega_{s}$. Nonetheless, when $\Omega_{p}=\Omega_{s}$, equation (2) reduces to equation (1) and is satisfied exactly. Thus, testing whether $\Omega_{s}=\Omega_{p}$ does not require any assumptions.

\section{NGC 2950}

An ideal target for this purpose is the S2B NGC 2950, which is a large $\left(2.7 \times 1{ }^{\prime} .8\right.$; de Vaucouleurs et al. 1991, hereafter RC3 $)$ and bright $\left(B_{T}=11.84 ; \mathrm{RC} 3\right)$ early-type barred galaxy. NGC 2950 is classified RSB0(r), and its total absolute magnitude is $M_{B_{T}}^{0}=-20.03$ (RC3), adopting a distance of $23.3 \mathrm{Mpc}$ (Tully 1988). The presence of a secondary stellar bar has been discussed extensively by Wozniak et al. (1995), Friedli et al. (1996), and Erwin \& Sparke (2002) on the basis of ground-based and Hubble Space Telescope images in both optical and near-infrared bandpasses. The secondary bar of NGC 2950 is typical of those in the samples of Laine et al. (2002) and of Erwin \& Sparke (2002). NGC 2950 meets all the requirements for the TW analysis: it has an intermediate inclination, and both bars have intermediate position angles (P.A.'s) between the major and minor axes of the disk and no evidence of spiral arms, patchy dust, or significant companions. 


\section{SURFACE PHOTOMETRY}

The photometric observations of NGC 2950 were carried out at the $1 \mathrm{~m}$ Jacobus Kapteyn Telescope on 2000 December 27-28. We took multiple exposures in the Harris $B(4 \times$ $1200 \mathrm{~s}), V(3 \times 480 \mathrm{~s})$, and $I(18 \times 150 \mathrm{~s})$ bandpasses using the SITe2 $2048 \times 2048 \mathrm{CCD}$. This camera has a scale of $0.33 \mathrm{pixel}^{-1}$, yielding an unvignetted field of view of $\sim 10^{\prime} \times$ $10^{\prime}$. The seeing FWHM was $\approx 1^{\prime \prime} .0$. The data reduction has been carried out using standard IRAF tasks as in Debattista, Corsini, \& Aguerri (2002, hereafter DCA02). Images were bias-subtracted, flat-fielded, cleaned of cosmic rays, and corrected for bad pixels. The sky-background level was removed by fitting a second-order polynomial to the regions free of sources. Photometric calibration, using standard stars, included corrections for atmospheric and Galactic extinction and for color as in DCA02.

The radial profiles of surface brightness, ellipticity, and P.A. were obtained by fitting elliptical isophotes with the IRAF task ELLIPSE. We first fitted ellipses, allowing their centers to vary to test for patchy dust obscuration. We found no evidence of a varying center within the errors of the fits and similar P.A. and ellipticity profiles for all bandpasses. Thus, we concluded that there is little or uniform obscuration, as required for the TW method. The ellipse fits were then repeated with the ellipse center fixed; the resulting photometric profiles are plotted in Figure 1. We interpreted the local maximum in ellipticity at $r \simeq 3^{\prime \prime}$ and the corresponding twist and stationary value in P.A. as the photometric signatures of the presence of a misaligned secondary bar inside the primary bar. This is confirmed by the analysis of $B-I$ and $V-I$ color maps and the unsharp mask of the original frames; in all, we find no evidence of other smallscale structures such as nuclear rings or disks, spiral arms, starforming regions, dust lanes, and/or dust patches, in agreement with previous results (Wozniak et al. 1995; Friedli et al. 1996; Erwin \& Sparke 2003). In particular, the structural details unveiled by the unsharp mask of the WFPC2/F814W image of the nucleus of NGC 2950 (Erwin \& Sparke 2003) are unlike those typical of nuclear stellar disks (Pizzella et al. 2002). The P.A.'s of the primary (P.A. ${ }_{p}=152^{\circ} .6 \pm 0.4$ ) and secondary bar (P.A. $=91.1 \pm 0.3$ ) were measured in the $I$-band image at $r \simeq 3^{\prime \prime}$ and $r \simeq 23^{\prime \prime}$, respectively, at the two peaks in the ellipticity profile (Fig. 1). The lengths of the primary $\left(a_{p}=\right.$ $34.3 \pm 2$ ". 1$)$ and secondary bar $\left(a_{s}=4 . .5 \pm 1.0\right)$ were measured using three independent methods based on Fourier amplitudes (Aguerri et al. 2000), Fourier and ellipse phases (DCA02), and a decomposition of the surface brightness profiles (Prieto et al. 2001). The inclination $\left(i=45^{\circ} .6 \pm 1.0\right)$ and P.A. of the disk (P.A. ${ }_{d}=116^{\circ} 1 \pm 1.0$ ) were determined by averaging the values measured between $65^{\prime \prime}$ and $100^{\prime \prime}$ in the $I$ band profile (Fig. 1).

\section{LONG-SLIT SPECTROSCOPY}

The spectroscopic observations of NGC 2950 were carried out at the $3.6 \mathrm{~m}$ Telescopio Nazionale Galileo on 2001 December 18 (run 1), 2002 March 20-22 (run 2), and 2003 March 9-11 (run 3). The Low Resolution Spectrograph is mounted in combination with the HR- $V$ grism No. 6 with 600 grooves $\mathrm{mm}^{-1}$ and a $0.7 \times 8$ '.1 slit. The detector was the Loral CCD with $2048 \times 2048$ pixels of $15 \times 15 \mu \mathrm{m}^{2}$. The wavelength range from 4660 to $6820 \AA$ was covered with a reciprocal dispersion of $1.054 \AA$ pixel $^{-1}$ and a spatial scale of $0^{\prime \prime} 275$ pixel $^{-1}$. We obtained four spectra with the slit along the disk major axis (runs 2 and 3) and 11 offset spectra with the slit parallel to it ( $Y=$

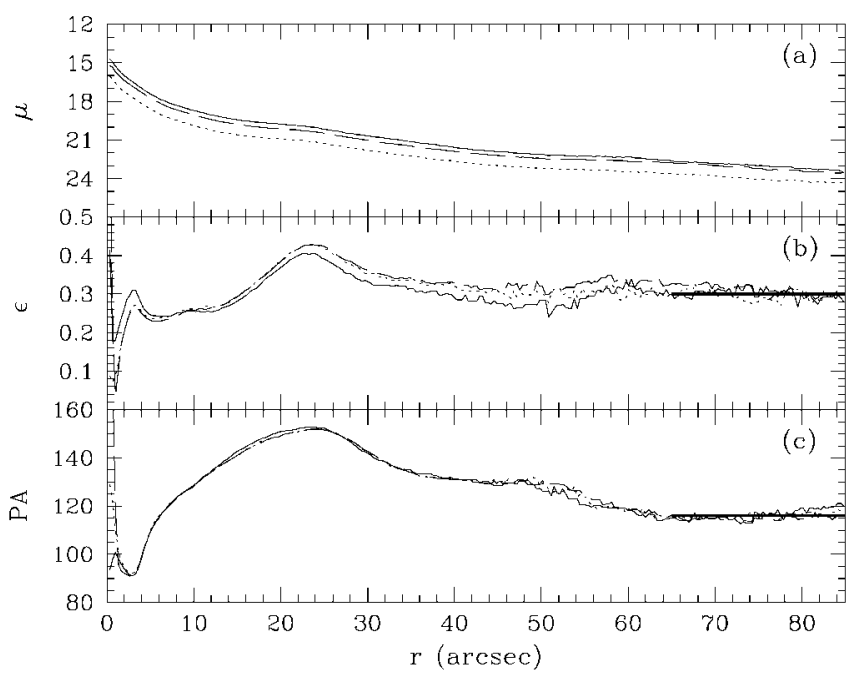

FIG. 1.-(a) Surface brightness, $\mu,(b)$ ellipticity, $\epsilon$, and (c) P.A. radial profiles of NGC 2950. The solid, dashed, and dotted lines refer to $I, V$, and $B$-band data, respectively. The thick lines represent the fits to the $I$-band $\epsilon$ and P.A. of the galaxy's disk.

$-3 " 1,+1 " .5,+2 " .8, \pm 10^{\prime \prime} 1$ in run $1, Y= \pm 5^{\prime \prime} .1, \pm 13^{\prime \prime} 4$ in run 2 , and $Y=-2$ ".5, +3.5 in run 3; Fig. 2). The exposure times were $2 \times 60$ and $2 \times 45$ minutes for the major-axis spectra obtained in runs 2 and 3, respectively, and 45 minutes for all the offset spectra. Comparison lamp exposures before and/or after each object integration ensured accurate wavelength calibrations. Spectra of $\mathrm{G}$ and $\mathrm{K}$ giant stars served as kinematical templates. The seeing FWHM was $\approx 1 " .2$ in run $1, \approx 1.5$ in run 2 , and $\approx 0.9$ in run 3 . Using standard MIDAS routines, all the spectra were bias-subtracted, flat-field-corrected, cleaned of cosmic rays, corrected for bad pixels, and wavelength-calibrated as in DCA02. The accuracy of the wavelength rebinning $\left(\$ 2 \mathrm{~km} \mathrm{~s}^{-1}\right)$ was checked by measuring wavelengths of the brightest night-sky emission lines. The instrumental resolution was $3.10 \AA$ (FWHM), corresponding to $\sigma_{\text {inst }} \approx 80 \mathrm{~km} \mathrm{~s}^{-1}$ at $5170 \AA$. The major-axis spectra obtained in the same run were co-added using the center of the stellar continuum as reference. In all the spectra, the contribution from the sky was determined by interpolating along the outermost $\approx 20^{\prime \prime}$ at the edges of the slit and then subtracted.

\section{PATTERN SPEEDS OF THE PRIMARY BAR AND SECONDARY BAR}

To measure $\mathcal{V}$ for each slit (Fig. $3 a$ ), we first collapsed each two-dimensional spectrum along its spatial direction in the wavelength range between 5060 and $5490 \AA$, obtaining a onedimensional spectrum. The value of $\mathcal{V}$ was then derived by fitting the resulting spectrum with the convolution of the spectrum of the K1 III star HR 4699 and a Gaussian line-of-sight velocity profile by means of the Fourier correlation quotient (FCQ) method (Bender 1990) as done in ADC03. We estimated uncertainties by Monte Carlo simulations with photon, readout, and sky noise. To compute $X$ for each slit (Fig. 3b), we extracted the luminosity profiles from the $V$-band image along the position of the slit after convolving the image to the seeing of the spectrum. The $V$-band profiles match very well the profiles obtained by collapsing the spectra along the wavelength direction, confirming that the slits were placed as intended. We used the $V$-band profiles to compute $\mathcal{X}$ because they are less noisy than those extracted from the spectra, particularly at large 


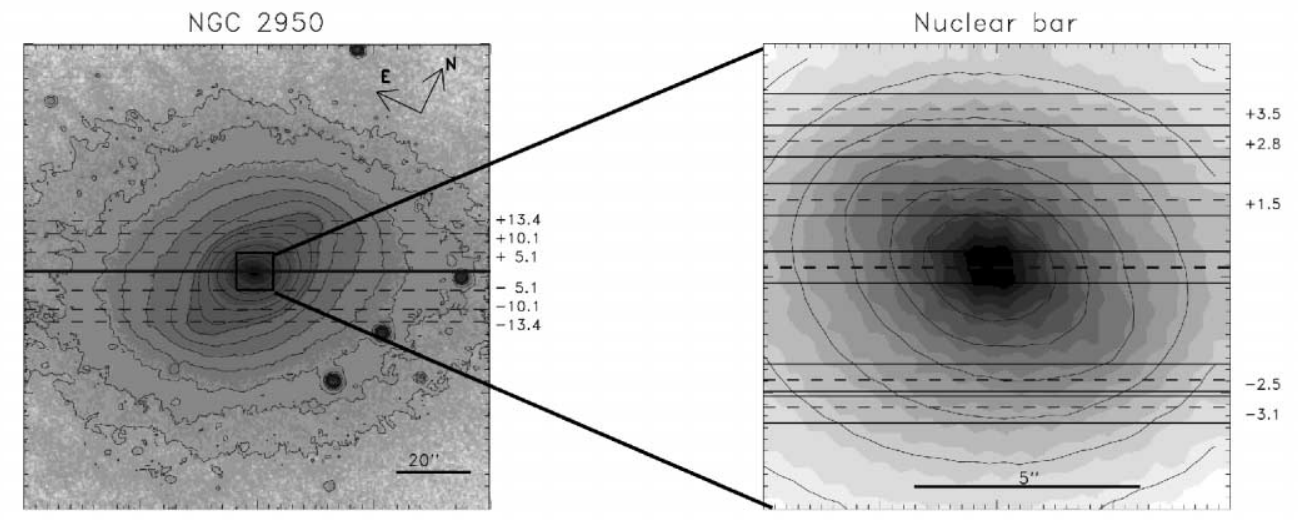

FIG. 2.-Left panel: Large-scale image of NGC 2950 showing the primary bar and disk with $I$-band contours and slit positions overlaid. The contours are spaced at $0.5 \mathrm{mag} \operatorname{arcsec}^{-2}$, with the innermost and outermost contours corresponding to $\mu_{I}=18.0$ and $\mu_{I}=24.0 \mathrm{mag} \operatorname{arcsec}^{-2}$, respectively. The solid and dashed lines correspond to the position of the spectra obtained along the disk major axis and at large offsets $\left(|Y| \geq 5^{\prime \prime} 1\right)$, respectively. For each slit position, the offset, $Y$, is given in arcseconds (we arbitrarily chose axes such that $Y$ increases from the southwest to the northeast side). Right panel: Zoom into the central region of NGC 2950 showing its secondary bar. $I$-band isocontours are spaced at $0.5 \mathrm{mag} \operatorname{arcsec}^{-2}$, with the innermost and outermost contours corresponding to $\mu_{I}=17.0$ and $\mu_{I}=19.5 \mathrm{mag} \operatorname{arcsec}^{-2}$, respectively. For each spectrum obtained at $|Y| \leq 3$ ".5, the solid and dashed lines mark the edges and the center of the slit, respectively.

radii. We obtained $\Omega_{p}$ from the values of $X$ and $\mathcal{V}$ for the slits at $|Y| \geq 3$ ".1 and at $Y=0^{\prime \prime}$ (the latter constrain only the zero point). Since the slits at $|Y| \geq 3$ 3.1 do not cross the secondary bar, we assume $X=\chi_{p}$ and $\mathcal{V}=\mathcal{V}_{p}$ for them and obtain $\Omega_{p} \sin i$ with a straight-line fit. This gives $\Omega_{p}=11.2 \pm 2.4 \mathrm{~km}$ $\mathrm{s}^{-1} \operatorname{arcsec}^{-1}\left(99.2 \pm 21.2 \mathrm{~km} \mathrm{~s}^{-1} \mathrm{kpc}^{-1}\right.$; Fig. 3c). The value of $\Omega_{p}$ does not change within errors $\left(\Omega_{p}=10.9 \pm 2.4 \mathrm{~km} \mathrm{~s}^{-1}\right.$ $\operatorname{arcsec}^{-1}$ ) when we exclude the slits at $Y=-3$ ".1, +3 ".5, confirming that they are not dominated by the secondary bar (Fig. 2).
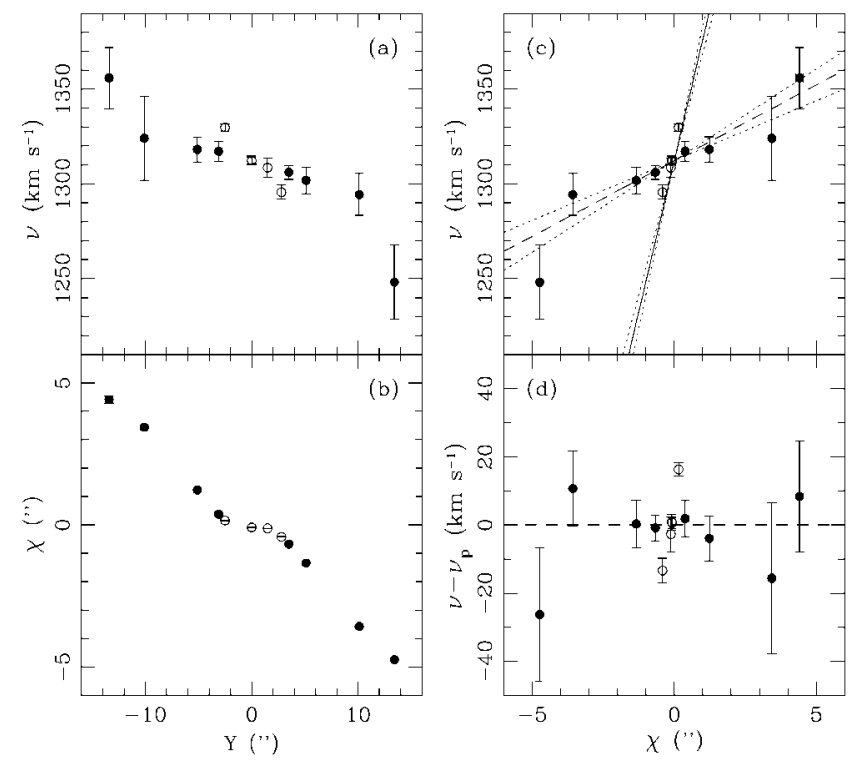

FIG. 3.-(a) Kinematic integrals $\mathcal{V}$ as a function of the slit offset $Y$ with respect to the major axis $\left(Y=0^{\prime \prime}\right)$. The open and filled circles correspond to slits crossing the secondary bar $\left(|Y| \leq 2^{\prime \prime} .8\right)$ and at $|Y| \geq 3$ ".1, respectively. (b) Photometric integrals $X$ as a function of the slit offset $Y$. (c) $\mathcal{V}$ as a function of $\mathcal{X}$ with different straight-line fits. These were obtained by including the slits at $Y=0^{\prime \prime}$ and only the slits at $|Y| \geq 3$ ".1 (dashed line, slope $\Omega_{p} \sin i=$ $8.0 \pm 1.7 \mathrm{~km} \mathrm{~s}^{-1} \operatorname{arcsec}^{-1}$ ) or the innermost slits at $|Y| \leq 2$ ".8 (solid line, slope $\left.\Omega_{s, 1} \sin i=63.7 \pm 7.1 \mathrm{~km} \mathrm{~s}^{-1} \operatorname{arcsec}^{-1}\right)$. The very different slopes of the two straight lines strongly suggest that the primary and secondary bars have different pattern speeds. (d) Residuals from the straight-line fit to the slits at $|Y| \geq 3 " .1$ and at $Y=0^{\prime \prime}$.
We used the FCQ to measure the line-of-sight velocity and the velocity dispersion profiles of the stellar component along the major axis (Fig. 4). All the major-axis spectra were coadded after being convolved to the same seeing. We derived the circular velocity in the disk region, $V_{c}=356_{-49}^{+61} \mathrm{~km} \mathrm{~s}^{-1}$, after a standard correction for the asymmetric drift as in $\mathrm{ADC03}$. Thus, the corotation radius of the primary bar is $D_{p}=V_{c} / \Omega_{p}=32^{\prime \prime} 4_{-6.2}^{+8.7}$, and the ratio $\mathcal{R}_{p} \equiv D_{p} / a_{p}$ of the corotation radius to the bar semimajor axis is $R_{p}=1.0_{-0.2}^{+0.3}$ (the error intervals on $D_{p}$ and $\mathcal{R}_{p}$ are at the $68 \%$ confidence level and were measured with Monte Carlo simulations as in ADC03). We conclude that, within the errors, the primary bar of NGC 2950 is consistent with all previous measurements of $R$ in SB0 galaxies (ADC03), which gives us confidence in our assumption that the signals in the outer slits are generated by the primary bar only.

The photometric and kinematic integrals measured with the innermost slits $(|Y| \leq 2$ ".8) include a contribution from the secondary bar. In particular, $|\mathcal{V}| \gg\left|\mathcal{V}_{p}\right|$ for the slits at $Y=$

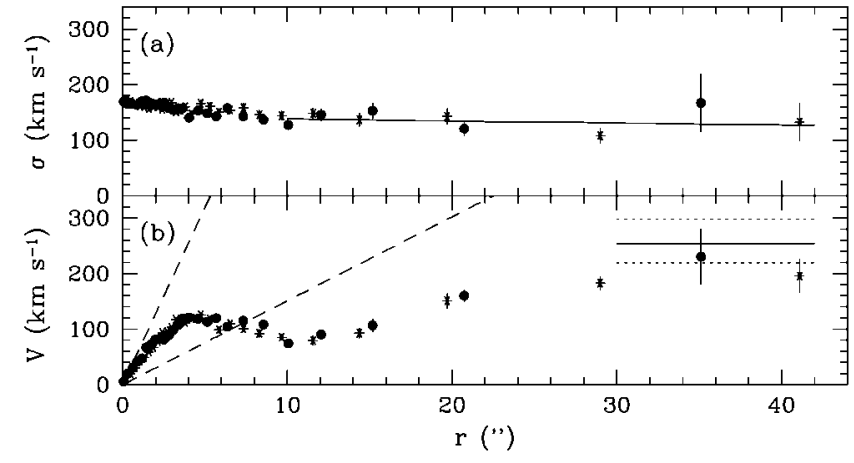

FIG. 4.- (a) Major-axis radial profile of the stellar line-of-sight velocity dispersion fitted with an exponential profile at $r \geq 10^{\prime \prime}$ (solid line). (b) Major-axis radial profile of the stellar line-of-sight velocity (after subtracting the systemic velocity $V_{\text {sys }}=1312 \pm 3 \mathrm{~km} \mathrm{~s}^{-1}$ ) and the $V_{c} \sin i$ curve (solid line) with errors (dotted lines) obtained by applying the asymmetric drift for $r \geq 30^{\prime \prime}$ as in ADC03. The dashed lines have slopes $\Omega_{s, 1} \sin i=63.7 \mathrm{~km} \mathrm{~s}^{-1} \operatorname{arcsec}^{-1}$ and $\left|\Omega_{s, 2}\right| \sin i=15.1 \mathrm{~km} \mathrm{~s}^{-1} \operatorname{arcsec}^{-1}$. In $(a)$ and $(b)$, the measured profiles are folded around the center, with the filled circles and asterisks referring to the southeast (receding) and northwest (approaching) sides, respectively. 
-2 ".5, +2 ".8 if we extrapolate $\mathcal{V}_{p}$ from large $|Y|$. A straightline fit to $\mathcal{X}$ and $\mathcal{V}$ for the slits at $|Y| \leq 2^{\prime \prime} .8$ has a slope $\left(=63.7 \pm 7.1 \mathrm{~km} \mathrm{~s}^{-1} \operatorname{arcsec}^{-1}\right.$; Fig. $\left.3 c\right)$ that is different at better than the $99 \%$ confidence level from the slope $(=8.0 \pm 1.7 \mathrm{~km}$ $\mathrm{s}^{-1} \operatorname{arcsec}^{-1}$, Fig. $3 c$ ) of the straight-line fit for the primary bar. This may be because $\Omega_{p} \neq \Omega_{s}$; however, to justify this conclusion, we needed to exclude the possibility of a systematic error due to P.A. errors, which $\chi$ and $\mathcal{V}$ are quite sensitive to (Debattista 2003). We tested whether the difference in the slopes of the straight-line fits may only be due to P.A. errors when $\Omega_{p}=\Omega_{s}$ by building a model of a S2B galaxy with a single pattern speed from an $N$-body simulation of an SB0 galaxy described in Debattista (2003). We rotated particles by $90^{\circ}$, rescaled their phase-space coordinates by a factor of 0.2 , and added them back to the original galaxy with various primary-to-secondary mass ratios to approximately match NGC 2950. After projecting this system as in NGC 2950, we proceeded to measure $\mathcal{X}$ and $\mathcal{V}$ for slits misaligned with the major axis. Even when P.A. errors reached $\pm 5^{\circ}$, we were not able to produce a system that approaches the behavior of our observations. In particular, we were not able to produce a system in which the slopes of the integrals plotted versus $|Y|$ are larger for $\mathcal{V}$ and smaller for $\mathcal{X}$ in the region of the secondary bar than in the region of the main bar, as we observed in NGC 2950 (Figs. 3a and 3b). We therefore concluded that the signatures we observed could not be an artifact of any P.A. misalignments on two bars rotating with a single pattern speed. Our results, therefore, lead us to conclude, directly and for the first time, that $\Omega_{s} \neq \Omega_{p}$.

However, estimating $\Omega_{s}$ is model-dependent; we illustrate this by considering two extreme cases. First, we assumed that the secondary bar dominates at $|Y| \leq 2$ ".8 and used equation (1) with $\Omega$ replaced by $\Omega_{s}$ to find $\Omega_{s, 1}=89.2 \pm 9.9 \mathrm{~km} \mathrm{~s}^{-1}$ $\operatorname{arcsec}^{-1}$ (Fig. 3c). In the second case, we rewrote equation (2) as $\chi_{s}\left(\Omega_{s}-\Omega_{p}\right) \sin i=\mathcal{V}-\Omega_{p} \chi \sin i$. The observed quanti- ties are $\chi$ and $\mathcal{V}$, while $\Omega_{p}$ was measured above. To obtain $\chi_{s}$, first we derived the values of $\chi_{p}$ in the region of the nuclear bar by fitting a straight line to the values of $\mathcal{X}$ at $|Y| \geq 10^{\prime \prime}$.1 (fits extending to smaller $|Y|$ give larger $\left|\Omega_{s}\right|$ ) and obtained $\chi_{s}=\chi-\chi_{p}$. Then plotting $\left(\mathcal{V}-\Omega_{p} \chi \sin i\right)$ versus $\chi_{s}$, we obtain $\left(\Omega_{s}-\Omega_{p}\right) \sin i$ as the slope of the best-fitting line; the result is $\Omega_{s, 2}=-21.2 \pm 6.2 \mathrm{~km} \mathrm{~s}^{-1} \operatorname{arcsec}^{-1}$; i.e., the secondary bar is counterrotating relative to the primary. (Note that the range from $\Omega_{s, 1}$ to $\Omega_{s, 2}$ passes smoothly through $\pm \infty$, i.e., a vertical line.)

\section{CONCLUSIONS}

We showed that the primary bar in NGC 2950 is rapidly rotating. If this is the norm in $\mathrm{S} 2 \mathrm{~B}$ galaxies, then it guarantees that primary bars are efficient at funnelling gas down to the radius of influence of secondary bars. In Figure 4, we plot the lines of slope $\Omega_{s, 1} \sin i$ and $\Omega_{s, 2} \sin i$. The range of $\Omega_{s}$ is large enough that it must include the case in which $\mathcal{R}_{s} \simeq 1$. However, it also includes the case in which $R_{s} \sim 2$, and hydrodynamical simulations find that this leads to inefficient gas transport (Maciejewski et al. 2002).

We suggest two avenues for fruitful future work. First, since the two bars cannot be in exact solid-body rotation (Louis \& Gerhard 1988; Maciejewski \& Sparke 2000; Rautiainen et al. 2002), a more accurate measurement of $\Omega_{s}$ will require careful modeling and comparison with simulations to account for such effects. Second, it may be that secondary bars oscillate about an orientation perpendicular to the primary bar, possibly accounting for $\Omega_{s}<0$. This can be tested by repeating our measurements on a sample of S2B galaxies. Nonetheless, we can confidently conclude that in NGC 2950, the two bars must have different pattern speeds, with the secondary bar having a larger pattern speed.

\section{REFERENCES}

Aguerri, J. A. L., Debattista, V. P., \& Corsini, E. M. 2003, MNRAS, 338, 465 (ADC03)

Aguerri, J. A. L., Muñoz-Tuñón, C., Varela, A. M., \& Prieto, M. 2000, A\&A, 361,841

Bender, R. 1990, A\&A, 229, 441

Buta, R., \& Crocker, D. A. 1993, AJ, 105, 1344

Debattista, V. P. 2003, MNRAS, 342, 1194

Debattista, V. P., Corsini, E. M., \& Aguerri, J. A. L. 2002, MNRAS, 332, 65 (DCA02)

de Vaucouleurs, G., de Vaucouleurs, A., Corwin, H. G., Jr., Buta, R. J., Paturel, G., \& Fouqué, P. 1991, Third Reference Catalogue of Bright Galaxies (New York: Springer) (RC3)

Erwin, P., \& Sparke, L. S. 2002, AJ, 124, 65 2003, ApJS, 146, 299

Eskridge, P. B., et al. 2000, AJ, 119, 536

Friedli, D. 1996, A\&A, 312, 761

Friedli, D., \& Martinet, L. 1993, A\&A, 277, 27

Friedli, D., Wozniak, H., Rieke, M., Martinet, L., \& Bratschi, P. 1996, A\&AS, 118,461
Knapen, J. H., Shlosman, I., \& Peletier, R. F. 2000, ApJ, 529, 93

Laine, S., Shlosman, I., Knapen, J. H., \& Peletier, R. F. 2002, ApJ, 567, 97

Louis, P. D., \& Gerhard, O. E. 1988, MNRAS, 233, 337

Maciejewski, W., \& Sparke, L. S. 2000, MNRAS, 313, 745

Maciejewski, W., Teuben, P. J., Sparke, L. S., \& Stone, J. M. 2002, MNRAS, 329,502

Pizzella, A., Corsini, E. M., Morelli, L., Sarzi, M., Scarlata, C., Stiavelli, M., \& Bertola, F. 2002, ApJ, 573, 131

Prieto, M., Aguerri, J. A. L., Varela, A. M., \& Muñoz-Tuñón, C. 2001, A\&A, 367, 405

Rautiainen, P., Salo, H., \& Laurikainen, E. 2002, MNRAS, 337, 1233

Regan, M. W., \& Mulchaey, J. S. 1999, AJ, 117, 2676

Sellwood, J. A., \& Merritt, D. 1994, ApJ, 425, 530

Tremaine, S., \& Weinberg, M. D. 1984, ApJ, 282, L5

Tully, R. B. 1988, Nearby Galaxy Catalog (Cambridge: Cambridge Univ. Press)

Wozniak, H., Friedli, D., Martinet, L., Martin, P., \& Bratschi, P. 1995, A\&AS, 111,115 